\title{
Physical Layer Security in Nonorthogonal Multiple Access Wireless Network with Jammer Selection
}

\author{
Langtao Hu (iD, Xin Zheng, and Chunsheng Chen \\ Anqing Normal University, AnQing 246133, China \\ Correspondence should be addressed to Langtao Hu; 122634998@qq.com
}

Received 1 April 2019; Accepted 22 October 2019; Published 16 November 2019

Academic Editor: Salvatore Sorce

Copyright (c) 2019 Langtao Hu et al. This is an open access article distributed under the Creative Commons Attribution License, which permits unrestricted use, distribution, and reproduction in any medium, provided the original work is properly cited.

\begin{abstract}
The physical layer security of downlink nonorthogonal multiple access (NOMA) network is analyzed. In order to improve the secrecy probability, friendly jammers are jointed in the NOMA network. Two jammer schemes are proposed in the NOMA network. All the jammers transmit jamming signal without jammer selection in the first scheme (NO JS scheme). Jammers are selected to transmit jamming signal if their interfering power on scheduled users is below a threshold in the second scheme (JS scheme). A stochastic geometry approach is applied to analyze the outage probability and the secrecy probability. Compared with the NO JS scheme and traditional scheme (without jointing jammers), the jammer selection scheme provides a good balance between the user outage probability and secrecy probability. Numerical results demonstrate that the security performance of the two proposed schemes can be improved by jointing the jammers in the NOMA wireless network.
\end{abstract}

\section{Introduction}

The development of mobile internet and Internet of Things brings lots of challenging requirements to fifth-generation $(5 \mathrm{G})$ networks, such as increasing high data rate and low latency [1-3]. The nonorthogonal multiple access has been considered as key promising multiple access candidates for $5 \mathrm{G}$ cellular networks. NOMA allows serving multiple users simultaneously using the same frequency/time resources at the cost of increased intracell interference. At the base station side, messages for multiple users are superposed by superposition coding. The successive interference cancellation (SIC) technique is applied to extract the intended message in the receiver side. Security is always an important issue for wireless networks, since the broadcast nature of wireless radio propagation makes it easily be overheard by eavesdroppers.

1.1. Related Works. Recently, NOMA has received remarkable attention both in the world of academia and industry [4-6]. Power-domain NOMA has been proposed for the 3GPP longterm evolution initiative in [4]. The authors in [5] investigated the performance of NOMA in a cellular downlink scenario with randomly deployed users. It was shown that NOMA can achieve superior ergodic sum rate performance than traditional orthogonal multiple access (OMA) counter parts. Challenges, opportunities, and future research trends for NOMA design are highlighted to provide some insight into the potential future work of researchers in [6]. The security performance of the NOMA networks can be improved by invoking the protected zone and by generating noise at the BS as shown by Liu et al. [7]. The physical layer security of uplink nonorthogonal multiple access is analyzed by Gerardo Gomez et al. [8]. Tao et al. [9] proposed a new reliable physical layer network coding and cascade-computation decoding scheme. The authors in [10] proposed an opportunistic multiple-jammer selection scheme for enhancing the physical layer security. The authors in [11] studied the security-reliability tradeoff analysis for conventional single-hop networks under a single passive eavesdropper attack. The authors in [12] enhanced physical layer security for downlink heterogeneous networks by using friendly jammers and full-duplex users. Recently, stochastic geometry has been used to model some network, such as largescale HET wireless networks [10], massive MIMO-enabled HetNets [14], cognitive cellular wireless networks [15], 
multitier millimeter wave cellular networks [16], and NOMA network [17]. It has been succeeded to develop tractable models to characterize and better understand the performance of these networks, and these models have been shown to provide tractable yet accurate performance bounds for these networks.

1.2. Motivations and Contributions. In this paper, we investigate the physical layer security of NOMA with friendly jammers and multiple noncolluding eavesdroppers (EVEs) in large-scale networks with stochastic geometry. For the sake of easy deployment, all nodes in NOMA wireless network are equipped with single antenna, and eavesdroppers are randomly distributed along the whole plane. The jammers are assumed to transmit friendly artificial noise. To enhance the physical layer security, two schemes are proposed with jointing jammers in NOMA network. In order to alleviate the interference from jammers to scheduled users, a jammer selection policy is discussed based on the received jamming power at the user. When the jammer power is below a threshold, the jammer around the scheduled user is active; otherwise, the jammer is idle. Unlike the scheme in $[7,8]$, the security performance of the NOMA networks can be improved by generating noise at the BS in [7], and physical layer security in uplink NOMA is discussed in [8]. In this paper, friendly jammers will be jointed, which can enhance the physical layer security in downlink NOMA wireless network. The main contributions of this paper are summarized as follows:

(1) Two physical layer security schemes are proposed, where friendly jammers are jointed in NOMA wireless network. Jammers transmit friendly artificial noise. A jammer selection policy is discussed based on the received jamming power at user.

(2) Using stochastic geometry tools, the downlink NOMA performance is analyzed in terms of outage probability and secrecy probability. In particular, the BSs and user positions are model Poisson point process (PPP) on a 2-D plane. The active jammer positions are model Poisson hole process.

(3) All the analytical results are validated by system-level simulations. Our proposed schemes provide a better security performance compared with the traditional scheme.

The rest of the paper is organized as follows. In Section 2, we introduce the system model. In Section 3, the outage probability performance of proposed schemes is investigated. In Section 4, the user secrecy probability performance of proposed schemes is investigated. In Section 5, numerical simulation and analysis are discussed to verify these results. A conclusion is drawn in Section 6.

Notation: the expectation of function $f(x)$ with respect to $x$ is denoted as $E[f(x)]$. Cumulative distribution function of $f(x)$ is denoted as $F_{f}()$. The Laplace transform of $f(x)$ is denoted by $L_{f}(s)$. An exponential distributed random variable with mean 1 is denoted by $x \sim \exp (1)$. Let $I_{1}$ be a set and $I_{2}$ be a subset of $I_{1}$; then, $I_{1} \backslash I_{2}$ denotes the set of elements of $I_{1}$ that do not belong to $I_{2}$.

\section{System Model}

In this paper, we consider a dense multicell NOMA downlink wireless network in presence of eavesdroppers (EVEs) as shown in Figure 1. All BSs users and EVEs are equipped with one antenna. The BS locations are distributed as an independent homogeneous $\operatorname{PPP} \Phi_{B}$ with density $\lambda_{B}$ in two-dimensional plane. Without loss of generality, the analysis is performed in a typical cell denoted as $B S_{0}$. Based on Slivnyak's Theorem, due to stationarity of $\Phi_{B}$, the typical cell can reflect the averaged performance of the entire system. The system assumes a frequency reuse factor 1 ; hence, the same frequency resources are used in all cells. The radio resources are partitioned into a number of subbands. We assume the bandwidth of each subband is normalized to 1 . The UE and EVE locations are distributed as an independent homogeneous PPP $\Phi_{U}$ and $\Phi_{E}$ with densities $\lambda_{U}$ and $\lambda_{E}$, respectively. In this paper, the NOMA group includes two users. Existing results have shown that the NOMA group with more than two UEs may provide a better performance gain [18]. In order to process the SIC easily, two user NOMA networks are more practical in the reality system. $U E_{1}$ and $U E_{2}$ consist of a NOMA group. We assume $\lambda_{U} \gg \lambda_{B}$ so that a sufficient number of UEs can always be found to form the NOMA group in each cell. To enhance the security performance, friendly jammers are jointed in NOMA network. In the selective jammer scheme, a jammer is selected to be active if its interference power to the scheduler user is below a threshold. Actually, the threshold is the jammer exclusion zone around the scheduler user, i.e., $\Phi_{J S}=\left\{j \mid j \in \Phi_{J}, P_{J} R_{j, U_{i}}^{-a}<P_{J} D^{-a}\right.$, $\left.\forall U_{i} \in \Phi_{U_{s}}\right\} . \Phi_{J S}$ is the Poisson hole process of active jammer. $\Phi_{U_{s}}$ denotes the point process of scheduled users. $\Phi_{U_{s}}$ is an inhomogeneous PPP, for which the density is $\lambda_{U_{s}}$. $R_{j, U_{i}}^{-a}$ is the distance between the jammer and scheduled users. $D$ is the exclusion zone radius. $P_{J}$ is denoted as the transmit power of the jammer. $a$ is the pathloss exponent. $P_{b}$ is the total power of BS on a subband in downlink NOMA. The allocated powers of $U E_{1}$ and $U E_{2}$ can be denoted as $P_{1}=\varepsilon P_{b}$ and $P_{2}=(1-\varepsilon) P_{b}$, respectively, where $\varepsilon \in(0,0.5)$ is a NOMA power allocation parameter. $U E_{1}$ is assumed to be with a better normalized channel gain. $U E_{2}$ is assumed to be with a worse normalized channel gain. The two users are selected randomly in NOMA network. $P_{J}$ denotes the power of jammer. BS-transmitted signals to UE1 and UE2 are expressed as $x_{1}$ and $x_{2}$, respectively. Since UE1 and UE2 form a NOMA group, $x_{1}$ and $x_{2}$ are encoded as the composite signal at the $B S_{0}[12]$ :

$$
\bar{x}=\sqrt{P_{1}} x_{1}+\sqrt{P_{2}} x_{2} .
$$

The received signal at $U E_{i}, i \in\{1,2\}$ can be expressed as

$$
y_{i}=\sqrt{h_{i} r_{i}^{-a}} \bar{x}+n_{i}
$$

where $h_{i}$ is the Rayleigh fading gain between $B S_{0}$ and $U E_{i}$, which follows an exponential distribution with mean 1 . All $h_{i}$ are assumed to be i.i.d. $r_{i}$ is the distance between $B S_{0}$ and $U E_{i} . n_{i}$ is the additive noise. 


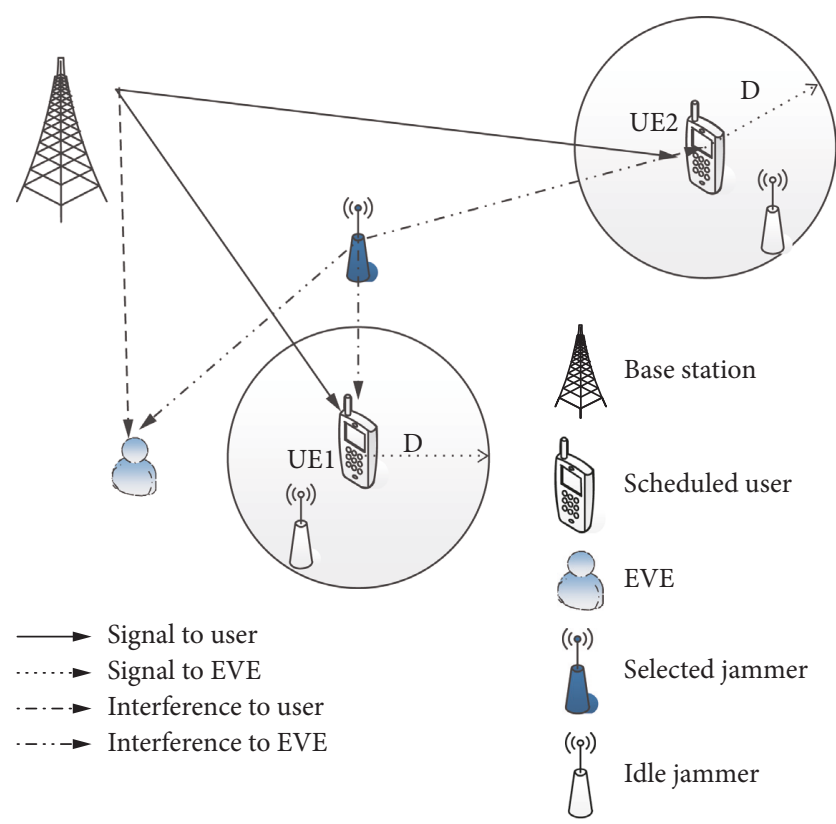

Figure 1: NOMA wireless network with the selected jammer.

\section{NOMA User Outage Probability}

We assume $U E_{1}$ has a better channel condition. At the receiver side, successive interference cancellation (SIC) is used to decode the intended message. $U E_{1}$ first decodes the $U E_{2}$ signal $x_{2}$ and removes it from the received composite signal; after that, $U E_{1}$ can further decode its signal $x_{1}$. $U E_{2}$ decodes $x_{2}$ directly by treating $x_{1}$ as interference. We consider the possible SIC error propagation, which is caused by decoding unsuccessfully in the first step, and thus, error is carried over to the next-level decoding. Let $\beta$ be the fraction of NOMA interference due to SIC error propagation. Noise can be safely neglected in a dense interference limited wireless system. The signal to interference ratio (SIR) of $U E_{1}$ can be represented as $\operatorname{SIR}_{U_{1}}=\left(\left(P_{1} h_{1} r_{1}^{-a}\right) /\left(I_{B_{1}}+I_{J S_{1}}+I_{W_{1}}\right)\right)$, where $I_{B_{1}}=\sum_{j \in \Phi_{B} \backslash B S_{0} P_{b} g_{1, j} R_{1, j}^{-a}}$ denotes the cumulative downlink intercell interference from the all other cells and $I_{J S_{1}}=\sum_{y \in \Phi_{I S} P_{j} h_{1, y} R_{1, y}^{-a}}$ denotes cumulative downlink interference from the selected jammers. $I_{W_{1}}=\beta P_{2} h_{1} r_{1}^{-a}$ denotes the interference from SIC error propagation. $g_{1, j}$ is the Rayleigh fading an exponential distribution with mean 1 , $g_{1, j} \sim \exp (1) . \Phi_{B} \backslash B S_{0}$ represents the set of all BSs excluding $B S_{0}$.

The achievable rate of $U E_{1}$ on each subband in NOMA network is given as

$$
\begin{aligned}
\tau_{1} & =\log \left(1+S I R_{U_{1}}\right) \\
& =\log \left(1+\frac{c_{1} P_{1}}{\beta c_{1} P_{2}+1}\right),
\end{aligned}
$$

where $c_{1}=\left(\left(h_{1} r_{1}^{-a}\right) /\left(I_{B_{1}}+I_{J S_{1}}\right)\right)$ is the $U E_{1}$ channel gain including pathloss and fast fading normalized by two kinds of interferences. The signal to interference ratio of $U E_{2}$ can be represented as $\operatorname{SIR}_{U_{2}}=\left(\left(P_{2} r_{2}^{-a} h_{2}\right) /\left(I_{B_{2}}+I_{J S_{2}}+P_{1} r_{2}^{-a} h_{2}\right)\right)$, where $I_{B_{2}}=\sum_{j \in \Phi_{B} \backslash B S_{0} P_{b} g_{2, j}{ }_{2, j}^{-a}}$ is the cumulative downlink intercell interference from all the other cells. $I_{J S_{2}}=\sum_{y \in \Phi_{J S} P_{J} h_{2, y} R_{2, y}^{-a}}$ denotes cumulative downlink interference from the selective jammers. $P_{1} r_{2}^{-a} h_{2}$ is the interference from $U E_{1}$.

The achievable rate of $U E_{2}$ on each subband in NOMA network is given as

$$
\begin{aligned}
\tau_{2} & =\log \left(1+\operatorname{SIR}_{U_{2}}\right) \\
& =\log \left(1+\frac{c_{2} P_{2}}{c_{2} P_{1}+1}\right),
\end{aligned}
$$

where $c_{2}=\left(\left(h_{2} r_{2}^{-a}\right) /\left(I_{B_{2}}+I_{J S_{2}}\right)\right)$. We assume that two users are randomly selected among all scheduled users. Two users are marked as $U E_{n}$ and $U E_{m}$. The normalized channel gains of $U E_{n}$ and $U E_{m}$ are denoted as $c_{n}$ and $c_{m}$, respectively. Let $U E_{1}=\left\{U E_{i} \mid U E_{i} \in\left\{U E_{n}, U E_{m}\right\}, c_{i}=\max \left(c_{n}, c_{m}\right)\right\}$, and $U$ $E_{2}=\left\{U E_{i} \mid U E_{i} \in\left\{U E_{n}, U E_{m}\right\}, c_{i}=\min \left(c_{n}, c_{m}\right)\right\}, \quad z=\max$ $(x, y)$, and $\Phi=\min (x, y)$. The CDF of $z$ and $\omega$ can be represented as $F_{z}(z)=F_{x y}(z, z) ; F_{\varpi}(\Phi)=F_{x}(\Phi)+F_{y}$ $(\bowtie)-F_{x y}(\omega, \oplus)[18]$. Thus, CDFs of $c_{1}$ and $c_{2}$ can be derived as follows:

$$
\begin{aligned}
F_{c_{1}}(C) & =F_{c_{n} c_{m}}(C, C)=F_{c}(C)^{2}, \\
F_{c_{2}}(C) & =F_{c_{n}}(C)+F_{c_{m}}(C)-F_{c_{n} c_{m}}(C, C) \\
& =2 F_{c}(C)-F_{c}(C)^{2},
\end{aligned}
$$

where $F_{c}(C)=1-P(c>C)$ and $P(c>C)$ denotes the probability of $c>C$. According to (5) and (6), $F_{c_{1}}(C)$ and $F_{c_{2}}(C)$ can be given as $(7)$ and $(8)$, respectively:

$$
\begin{aligned}
F_{c_{1}}(C) & =F_{c}(C)^{2}=(1-P(c>C))^{2}, \\
F_{c_{2}}(C) & =2 F_{c}(C)-F_{c}(C)^{2} \\
& =2(1-P(c>C))-(1-P(c>C))^{2} .
\end{aligned}
$$

For a given normalized channel gain to $U E_{1}$ or $U E_{2}, c=$ $\left(\left(h_{i} r_{i}^{-a}\right) /\left(I_{B}+I_{J S}\right)\right)$ and $P(c>C)$ can be derived as follows:

$$
\begin{aligned}
P(c>C) & =\mathrm{E}_{r_{i}}\left[P(c>C) \mid r_{i}\right] \\
& =\int_{r_{i}>0} P\left(\frac{h_{i} r_{i}^{-\alpha}}{I_{B}+I_{J S}}>C \mid r_{i}\right) f\left(r_{i}\right) \mathrm{d} r_{i} \\
& =\int_{r_{i}>0} E_{I_{B}, I_{J S}}\left[\exp \left(-C r_{i}^{\alpha}\left(I_{B}+I_{J S}\right)\right)\right] f\left(r_{i}\right) \mathrm{d} r_{i} \\
& =\int_{r_{i}>0} L_{I_{B}}\left(C r_{i}^{\alpha}\right) L_{I_{J S}}\left(C r_{i}^{\alpha}\right) f\left(r_{i}\right) \mathrm{d} r_{i} .
\end{aligned}
$$

In (9), the second term follows from $h_{i} \sim \exp (1)$ [13]. The Laplace transform of $I_{B}$ is given by 


$$
\begin{aligned}
L_{I_{B}}(s) & =E_{\Phi_{B}, g}\left[-\exp \left(-s \sum_{j \in \Phi_{B} / B S 0} g_{i, j} R_{j}^{-a} P_{B}\right)\right] \\
& =\exp \left(-2 \pi \lambda_{B} \int_{r_{i}}^{\infty}\left(1-\frac{1}{1+s v^{-a} P_{B}}\right) v \mathrm{~d} v\right) \\
& =\exp \left(-2 \pi \lambda_{B}\left(s P_{B}\right)^{\delta} \int_{r_{i}^{2} /\left(s P_{B}\right)^{\delta}}^{\infty} \frac{1}{1+u^{\delta}} \mathrm{d} u\right),
\end{aligned}
$$

where $\delta=2 / a$. The Laplace transform of $I_{J S}$ is given by

$$
\begin{aligned}
L_{I_{J S}}(s) & =\mathrm{E}_{\Phi_{J}, h}\left[-\exp \left(-s \sum_{j \in \Phi_{J S}} h_{i, j} R_{i, j}^{-a} P_{J}\right)\right] \\
& =\mathrm{E}_{\Phi_{J}}\left(\prod_{j \in \Phi_{J} \backslash B\left(U E_{i}, D\right)} L_{h_{i, j}}\left(s R_{i, j}^{-a} P_{J}\right)\right) \\
& \stackrel{(a)}{=} \mathrm{E}_{\Phi_{J}}\left(\exp \left(-\lambda_{J} \int_{j \in \Phi_{J} \backslash B\left(U E_{i}, D\right)}\left(1-L_{h_{i, j}}\left(s P_{J} x^{-a}\right) \mathrm{d} x\right)\right)\right) \\
& \stackrel{(b)}{\approx} \exp \left(-2 \pi \lambda_{J} D^{2-a} P_{J} s(a-2)^{-1}{ }_{2} F_{1}\right. \\
& \left.\cdot\left(1,1-\frac{2}{a} ; 2-\frac{2}{a} ;-\frac{s P_{J}}{D^{a}}\right)\right) .
\end{aligned}
$$

In step (a), $B\left(U E_{i}, D\right)$ is a hole of radius $D$ centered at $U E_{i}, \Phi_{J} \backslash B\left(U E_{i}, D\right)$ denotes the active jammers location, and $\Phi_{J}$ is the jammer baseline PPP from which the hole is carved out. In step (b), we only discuss the hole around the $U E_{i}$. The step (b) follows from the probability generating functional of PPP [13], where ${ }_{2} F_{1}(a, b ; c ; d)$ is the Gauss hypergeometric function.

When all jammers are active without jammer selection (NO JS scheme), $\delta=(2 / a) . L_{I_{\text {NOIS }}}(s)$ denotes the Laplace transform of interference from all jammers without selection to $U E_{i}$ in NO JS scheme. We can derive $L_{I_{\text {NOJS }}}(s)$ as [13]

$$
L_{I_{\mathrm{NOJS}}}(s)=\exp \left(-\pi \lambda_{J} \frac{\left(s P_{J}\right)^{\delta}}{\sin c(\delta)}\right) \text {. }
$$

By plugging (10) and (11) into (9), $P(c>C$ ) with jammer selection in NOMA network is given as

$$
\begin{aligned}
P(c>C)= & \int_{r_{i}>0} \exp \left(-2 \pi \lambda_{B}\left(s P_{B}\right)^{\delta} \int_{r_{i}^{2} /\left(s P_{B}\right)^{\delta}}^{\infty} \frac{1}{1+u^{\delta}} \mathrm{d} u\right) \\
& \times \exp \left(-2 \pi \lambda_{J} D^{2-a} P_{J} s(a-2)^{-1} \times{ }_{2} F_{1}\right. \\
& \left.\cdot\left(1,1-\frac{2}{a} ; 2-\frac{2}{a} ;-\frac{s P_{J}}{D^{a}}\right)\right) \\
& \times\left. 2 \pi \lambda_{B} r_{i} \exp \left(-\pi \lambda_{B} r_{i}^{2}\right) \mathrm{d} r_{i}\right|_{s=C r_{i}^{\alpha}} .
\end{aligned}
$$

When $a=4$,

$$
\begin{aligned}
P(c>C)= & \int_{r_{i}>0} \exp \%\left[-2 \pi \lambda_{B} r_{i}^{2}\left(C P_{B}\right)^{1 / 2}\right. \\
& \left.\cdot\left(\frac{\pi}{2}-\arctan \left(\frac{1}{\left(C P_{B}\right)^{1 / 2}}\right)\right) \%\right] \\
& \times \exp \left(-\pi \lambda_{J} D^{-2} P_{J} C r_{i 2}^{4} F_{1}\left(1,0.5 ; 1.5 ;-\frac{C r_{i}^{4} P_{J}}{D^{4}}\right)\right) \\
& \times 2 \pi \lambda_{B} r_{i} \exp \left(-\pi \lambda_{B} r_{i}^{2}\right) \mathrm{d} r_{i} .
\end{aligned}
$$

The outage probability of UE1 with jammer selection in NOMA network can be evaluated as follows:

$$
\begin{aligned}
P_{1}\left(\bar{\tau}_{1}, \bar{\tau}_{2}\right)= & 1-P\left(\tau_{1}>\bar{\tau}_{1}, \tau_{1 \longrightarrow 2}>\bar{\tau}_{2}\right) \\
& =1-P\left(\frac{c_{1} P_{1}}{\beta c_{1} P_{2}}>\gamma_{1}, \frac{c_{1} P_{2}}{c_{1} P_{1}+1}>\gamma_{2}\right) \\
& = \begin{cases}1 ; & \text { if } \gamma_{1} \geq \frac{P_{1}}{\beta P_{2}} \text { or } \gamma_{2} \geq \frac{P_{2}}{P_{1}}, \\
F_{c_{1}}\left(\max \left(\theta_{1}, \theta_{2}\right)\right) ; & \text { otherwise, }\end{cases}
\end{aligned}
$$

where $P\left(\tau_{1}>\bar{\tau}_{1}, \tau_{1 \longrightarrow 2}>\bar{\tau}_{2}\right)$ denotes the connection probability of $U E_{1}, \gamma_{1}$ and $\gamma_{2}$ denote the SIRs of $U E_{1}$ and $U E_{2}$, respectively. $\gamma_{1}=2^{\bar{\tau}_{1}}-1$ and $\gamma_{2}=2^{\bar{\tau}_{2}}-1 . \bar{\tau}_{1}$ and $\bar{\tau}_{2}$ are the target rate thresholds for $U E_{1}$ and $U E_{2}$, respectively. $\tau_{1 \longrightarrow 2}$ is the rate of decoding $U E_{2}$ signal $x_{2}$ in the $U E_{1}$ receiver, which must be greater than the QoS requirement of $U E_{2}, \tau_{1 \longrightarrow 2}>\bar{\tau}_{2}$ can ensure that $U E_{1}$ is able to remove $U E_{2}$ 's signal from interference.

$\theta_{1}=\left(\gamma_{1} /\left(p_{1}-\gamma_{1} \beta P_{2}\right)\right)$, and $\theta_{2}=\left(\gamma_{2} /\left(p_{2}-\gamma_{2} P_{1}\right)\right)$. Plugging $\max \left(\theta_{1}, \theta_{2}\right)$ into $(7)$, we get $F_{c_{1}}\left(\max \left(\theta_{1}, \theta_{2}\right)\right)$ in (15).

The outage probability of $U E_{2}$ with jammer selection in NOMA network can be evaluated as follows:

$$
P_{2}\left(\tau_{2} \leq \bar{\tau}_{2}\right)= \begin{cases}0 ; & \text { if } \gamma_{2} \geq \frac{P_{2}}{P_{1}} \\ F_{c_{2}}\left(\theta_{2}\right) ; & \text { otherwise. }\end{cases}
$$

Taking $\theta_{2}$ into (8), we can easily get $F_{c_{2}}\left(\theta_{2}\right)$ as shown in (16).

\section{NOMA User Secrecy Probability}

We investigate the secrecy probability of a randomly located NOMA user. In this work, the user secrecy probability corresponds to the probability that a secret message for the schedule user cannot be decoded by any noncolluding eavesdroppers, because $U E_{1}$ 's and $U E_{2}$ 's signals are derived by eavesdroppers in the similar way. Here, we only discuss the secrecy performance of $U E_{1}$. Hence, the secrecy probability can be expressed as 


$$
\begin{aligned}
P_{\mathrm{Sec}}\left(\gamma_{s}\right) & =P\left(\max _{x \in \Phi_{e}} \operatorname{SIR}(x)<\gamma_{s}\right) \\
& =P\left(\bigcap_{x \in \Phi_{e}} \operatorname{SIR}(x)<\gamma_{s}\right) \\
& =E\left(\prod_{x \in \Phi_{e}} P\left(\operatorname{SIR}(x)<\gamma_{s} \mid x\right)\right) \\
& =E_{\Phi_{e}}\left(\prod_{x \in \Phi_{e}}\left(1-\exp \left(-P_{1}^{-1} \gamma_{s} r_{x}^{a} I_{E}(x)\right)\right)\right) \\
& =\exp \left(-2 \pi \lambda_{e} \int_{0}^{\infty} L_{I_{E}(x)}\left(P_{1}^{-1} \gamma_{s} r_{x}^{a}\right) r_{x} \mathrm{~d} r_{x}\right),
\end{aligned}
$$

where $\operatorname{SIR}(x)$ of the typical EVE is given by

$$
\operatorname{SIR}(x)=\frac{P_{1} h_{x} r_{x}^{-a}}{I_{B \longrightarrow E}+I_{J S \longrightarrow E}},
$$

where $\gamma_{s}$ is the target secrecy SIR, $h_{x}$ is the Rayleigh fading gain between $B S_{0}$ and EVE, and $h_{x}$ follows an exponential distribution with mean 1. $r_{x}$ is the distance between $B S_{0}$ and EVE. $\quad L_{I_{E}}(s)=L_{I_{B \rightarrow E}}(s) L_{I_{I S \rightarrow E}}(s)$, where $L_{I_{B \rightarrow E}}(s)$ and $L_{I_{I S \rightarrow E}}(s)$ denote the Laplace transform of $I_{B \longrightarrow E}$ and $I_{J S \longrightarrow E}$, respectively. $I_{B \longrightarrow E}$ is the interference from the other cell BSs to EVE. $I_{J S \longrightarrow E}$ is the interference from the selective jammers to EVE. The detailed derivation of $L_{I_{I S \rightarrow E}}(s)$ is provided in Appendix A. We can derive the following:

$$
\begin{aligned}
L_{I_{E}}(s)= & L_{I_{B} \rightarrow E}(s) L_{I_{J S \rightarrow E}}(s) \\
= & \exp \left(-\frac{\pi s^{2} P_{1}^{\delta} \lambda_{B}}{\sin c(\delta)}\right) \exp \left(-\pi \lambda_{J} \frac{\left(s P_{J}\right)^{\delta}}{\sin c(\delta)}\right) \\
& \times \int_{0}^{\infty} H(v, s) g(v) \mathrm{d} v .
\end{aligned}
$$

Inserting (19) into (17), we can easily have the expression of $P_{\mathrm{Sec}}\left(\gamma_{s}\right)$.

When all jammers are active without jammer selection, $L_{I_{E}}(s)$ is given by

$$
\begin{aligned}
L_{I_{E}}(s) & =L_{I_{B \rightarrow E}}(s) L_{I_{J \rightarrow E}}(s) \\
& =\exp \left(-\pi \lambda_{B} \frac{\left(s P_{1}\right)^{\delta}}{\sin c(\delta)}\right) \exp \left(-\pi \lambda_{J} \frac{\left(s P_{J}\right)^{\delta}}{\sin c(\delta)}\right) .
\end{aligned}
$$

\section{Simulation Results and Discussion}

In this section, we present Monte Carlo simulations to evaluate the performance of the proposed scheme, and analytical results are illustrated and validated with extensive simulations in NOMA network. The default parameters are listed in Table 1 unless otherwise stated.

Our first proposed scheme is that all jammers are active without jammer selection in NOMA network, which is marked as "NO JS scheme." Our second proposed
TABle 1: Parameter assumptions.

\begin{tabular}{lcc}
\hline Parameter & Meaning & Default value \\
\hline$\lambda_{B}$ & Density of BS & $1 / \mathrm{km}^{2}$ \\
$\lambda_{J}$ & Density of jammer & $10 / \mathrm{km}^{2}$ \\
$\lambda_{E}$ & Density of eavesdropper & $11 / \mathrm{km}^{2}$ \\
$\lambda_{U}$ & Density of user & $100 / \mathrm{km}^{2}$ \\
$P_{B}$ & Transmission power of BS & $46 \mathrm{dBm}$ \\
$P_{J}$ & Transmission power of jammer & $23 \mathrm{dBm}$ \\
$\varepsilon$ & NOMA power allocation parameter & 0.3 \\
$\alpha$ & Pathloss exponent & 4 \\
$D$ & Jammer exclusion circle radius & $0.1 \mathrm{~km}$ \\
\hline
\end{tabular}

scheme is that some jammers are active with jammer selection in NOMA network, which is marked "JS scheme." There are no jammers in the traditional scheme in NOMA network. As expected, our proposed schemes provide a better security performance compared with the traditional scheme. The JS scheme provides a good balance between the user outage probability and the user secrecy probability. Figure 2 shows $U E_{1}$ outage probability $P_{1}\left(\bar{\tau}_{1}, \bar{\tau}_{2}\right)$ and $U E_{2}$ outage probability $P_{2}\left(\tau_{2} \leq \bar{\tau}_{2}\right)$ versus different target rates $\bar{\tau}_{1}$, where $\bar{\tau}_{2}$ is fixed to $0.1 \mathrm{bits} / \mathrm{s} /$ subband.

In Figure 2, "ana" (the dashed curves) and "sim" (the curves with circle marks) are the abbreviation of analysis and simulation, respectively. "UE1 NO JS ana" denotes the analytical result of UE1 outage probability using the first proposed scheme, where all jammers are active. "UE1 NO JS sim" denotes the simulation result of UE1 outage probability. "UE1 JS ana" denotes the analytical result of UE1 in the second proposed scheme, where some selective jammers are active. From Figure 2, we can observe that the analytical results match well with the simulation results, which validate the accuracy of the analysis.

In Figure 2, all jammers are active in the first proposed scheme. Jammers transmit artificial noise to legitimate NOMA users and the eavesdroppers. While transmitting noise to eavesdroppers, the jammer also transmits artificial noise to legitimate NOMA users in the proposed scheme. The noise is the interference for NOMA users. When the interference increases, the SIR decreases. Compared with the traditional scheme without jointing the jammer, the outage probability increases in our proposed scheme with jointing jammers in NOMA network. Compared to the first scheme, the outage probability decreases for the selective jammer scheme. In other words, the user connective performance improves. In Figure 2, we can see that $P_{1}\left(\bar{\tau}_{1}, \bar{\tau}_{2}\right)$ remains constant when $\bar{\tau}_{1} \leq 0.04 \mathrm{bit} / \mathrm{s} / \mathrm{subband}$. This is due to the fact that $U E_{1}$ needs to decode the signal intended to $U E_{2}$ first before it can decode the signal for itself. When $\bar{\tau}_{1}$ is below 0.04 , the outage is always remained by failing to decode $U E_{2}$ signal. The result also can be explained by the definition of outage probability of $U E_{1} P_{1}\left(\bar{\tau}_{1}, \bar{\tau}_{2}\right)=1-P_{1}\left(\tau_{1}>\bar{\tau}_{1}\right.$, $\left.\tau_{1 \rightarrow 2}>\bar{\tau}_{2}\right) ; P_{1}\left(\bar{\tau}_{1}, \bar{\tau}_{2}\right)$ is related to $\bar{\tau}_{1}$ and $\bar{\tau}_{2}$. If $\bar{\tau}_{2}$ is fixed, when $\bar{\tau}_{1}$ is quite small, $\tau_{1 \longrightarrow 2}>\bar{\tau}_{2}$ can guarantee $\tau_{1}>\bar{\tau}_{1}$ and $P_{1}\left(\tau_{1}>\bar{\tau}_{1}, \tau_{1 \longrightarrow 2}>\bar{\tau}_{2}\right) \quad$ becomes $\quad P_{1}\left(\tau_{1 \longrightarrow 2}>\bar{\tau}_{2}\right)$, so $P_{1}\left(\bar{\tau}_{1}, \bar{\tau}_{2}\right)$ is not a function of $\bar{\tau}_{1}$ and it remains constant 


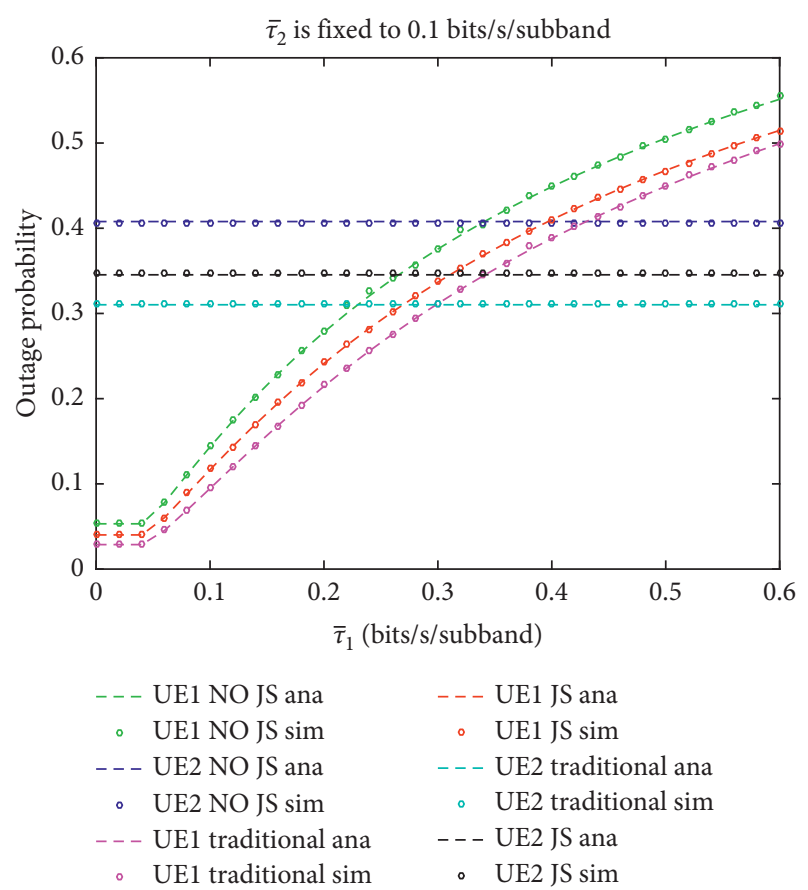

FIGURE 2: Outage probability of NOMA when $\bar{\tau}_{2}$ is fixed to 0.1 bits/ s/subband.

when $\bar{\tau}_{1}$ is quite small. When $\bar{\tau}_{1}>0.04$, the outage probability reduces as $\bar{\tau}_{1}$ increases. $P_{2}\left(\tau_{2} \leq \bar{\tau}_{2}\right)$ remains constant as $\bar{\tau}_{1}$ increases due to the fact that $P_{2}\left(\tau_{2} \leq \bar{\tau}_{2}\right)$ is not a function of $\bar{\tau}_{1}$.

Figure 3 shows the outage probability of $U E_{1}$ and $U E_{2}$ when $\tau_{1}$ is fixed to $\tau_{1}=0.1 \mathrm{bit} / \mathrm{s} / \mathrm{subband}$. We can observe $P_{1}\left(\bar{\tau}_{1}, \bar{\tau}_{2}\right)$ remains constant at first and then increases in the same way as shown in Figure 2. When $\bar{\tau}_{2}$ is small, $P_{1}\left(\bar{\tau}_{1}, \bar{\tau}_{2}\right)$ is only a function of $\bar{\tau}_{1}$, which is not affected by $\bar{\tau}_{2}$. As $\bar{\tau}_{2}$ continues to increase, both $\bar{\tau}_{1}$ and $\bar{\tau}_{2}$ will affect $P_{1}\left(\bar{\tau}_{1}, \bar{\tau}_{2}\right)$.

Figure 4 shows that outage probability of $U E_{1}$ in three schemes versus $\bar{\tau}_{1}$ with different $\varepsilon$ in NOMA network. The allocation power of $U E_{1}$ increases as $\varepsilon$ increases; we can see that while $\varepsilon$ increases in these schemes; $P_{1}\left(\bar{\tau}_{1}, \bar{\tau}_{2}\right)$ does not necessarily decrease. This outcome can be explained that more transmit power allocated to $U E_{1}$ also means less power allocated to $U E_{2}$, so it is difficult for $U E_{1}$ to decode $U E_{2}$ signal $x_{2}$. But the lower bound of outage probability of $U E_{1}$ can be improved, which is related by successfully decoding $U E_{2}$ 's signal $x_{2}$.

Figure 5 shows that $U E_{2}$ outage probability of three schemes versus $\bar{\tau}_{2}$ with different $\varepsilon$ in NOMA network. We can observe that the outage probability decreases whsen $\varepsilon$ is decreasing from 0.5 to 0.3 . This is because more power allocated to $U E_{2}$ will result in a better outage performance of $U E_{2}$.

Figure 6 shows the impact of imperfect SIC of three schemes with different $\beta$. From the SIR of $U E_{1}$, $\left(\left(c_{1} P_{1}\right) /\left(\beta c_{1} P_{2}+1\right)\right)$, we can see SIR decreases as $\beta$ increases. When $\beta=0.02$, compared to perfect SIC $\beta=0$, the outage probability of $U E_{1}$ increases.

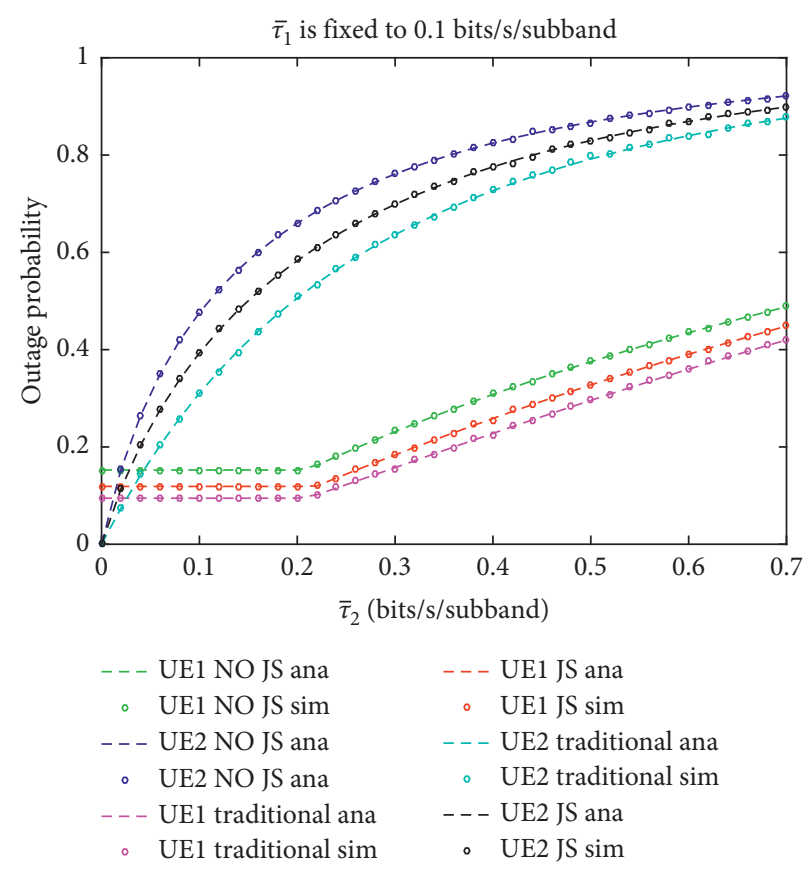

Figure 3: Outage probability of NOMA when $\bar{\tau}_{1}$ is fixed to 0.1 bits/ s/subband.

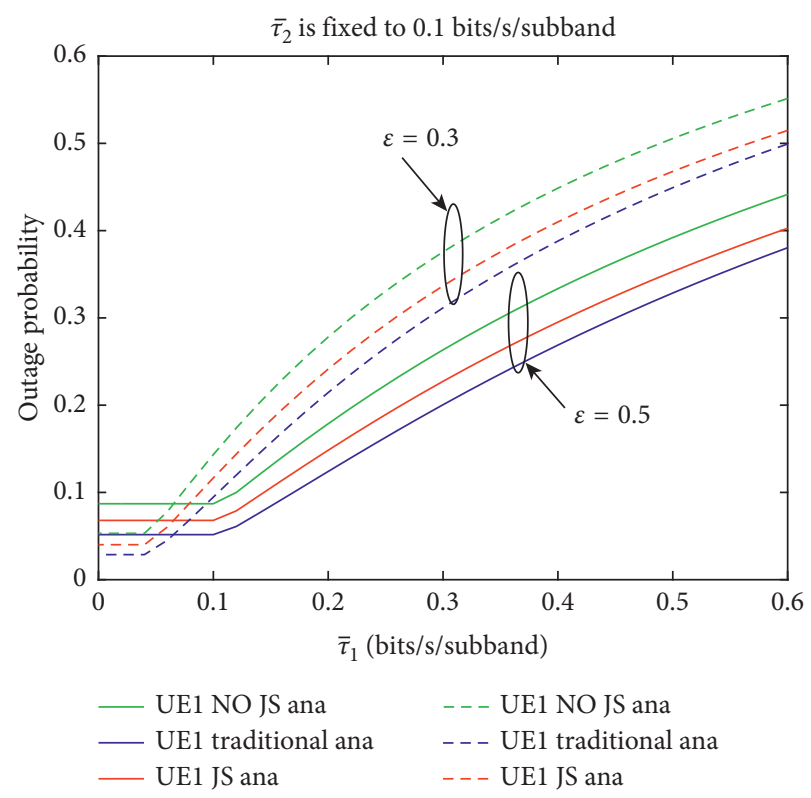

FIGURE 4: Outage probability of $U E_{1}$ three schemes versus $\bar{\tau}_{1}$ with different $\varepsilon$ in NOMA network.

Figure 7 shows that the $U E_{1}$ secrecy probability of our proposed scheme versus SIR threshold $\gamma_{s}$ with different $D$ exclusion zone radii. We can see that our proposed scheme has a better performance than the traditional scheme in secrecy probability in NOMA network. "NO JS scheme" will obtain the highest secrecy probability. But the outage probability of "NO JS scheme" is the worst. Secrecy probability of "JS scheme" with small jammer 


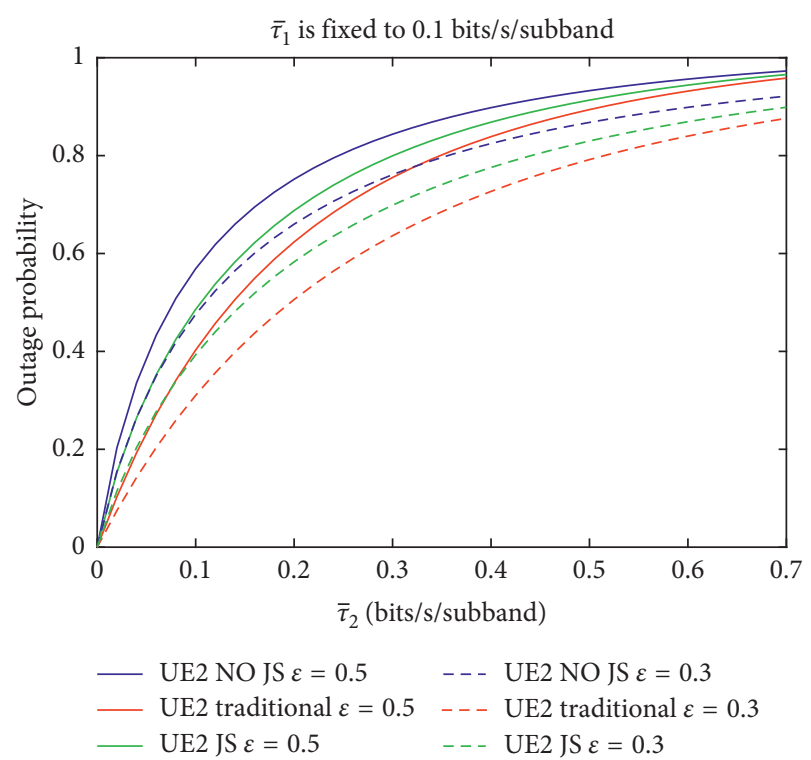

FIGURE 5: Outage probability of $U E_{2}$ in three schemes versus $\bar{\tau}_{2}$ with different $\varepsilon$ in NOMA network.

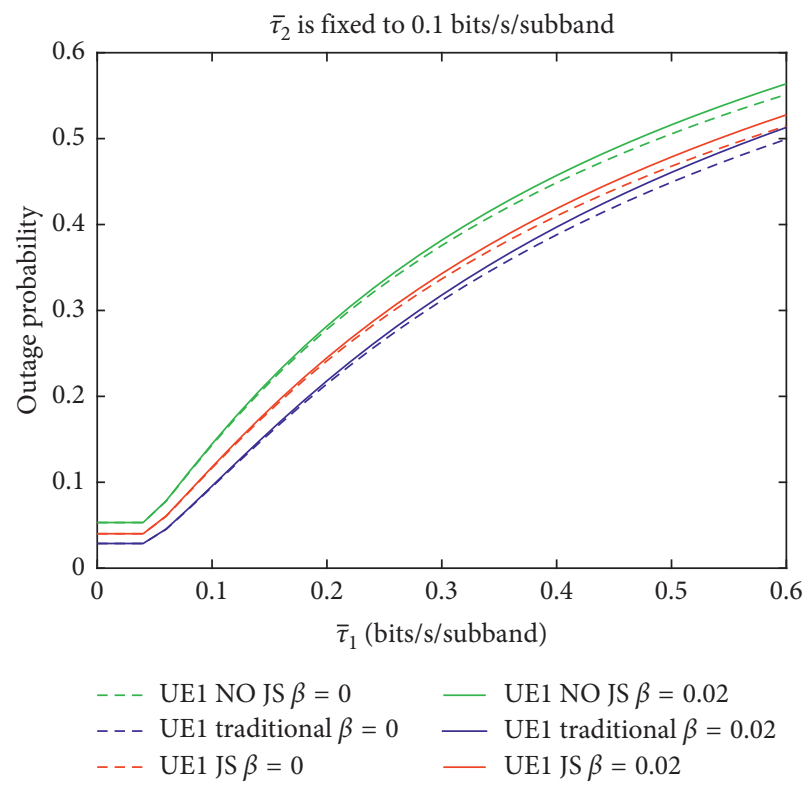

FIGURE 6: The impact of imperfect SIC of three schemes on NOMA with different $\beta$.

exclusion zone is better. When the exclusion zone radius increases, the small jammers transmit noise to interfere the eavesdropper, so secrecy probability decrease. Our proposed jammer selection scheme provides a good balance between the user outage probability and secrecy probability.

Figure 8 compares the connection probability performance of $U E_{1}$ versus jammer density $\lambda_{J}$. We can observe that simply deploying more jammers cannot enhance the connection probability. When jammer density $\lambda_{J}$ increases, the connection probability of JS scheme increases. But the connection probability of NO JS scheme

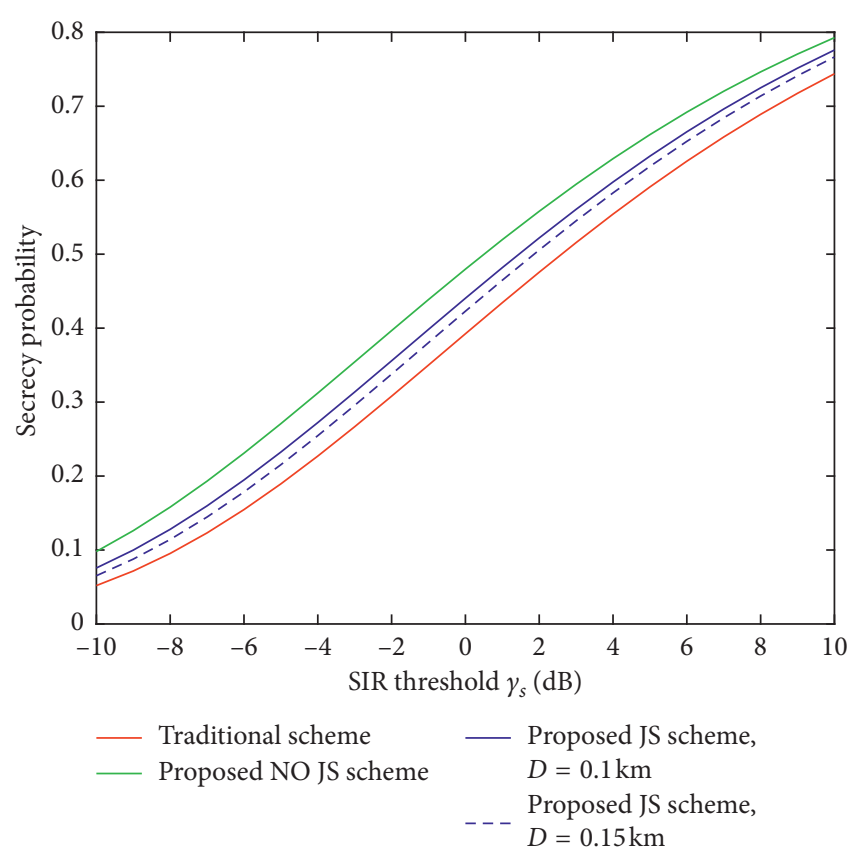

FIgURE 7: The UE1 secrecy probability versus SIR threshold $\gamma_{s}$.

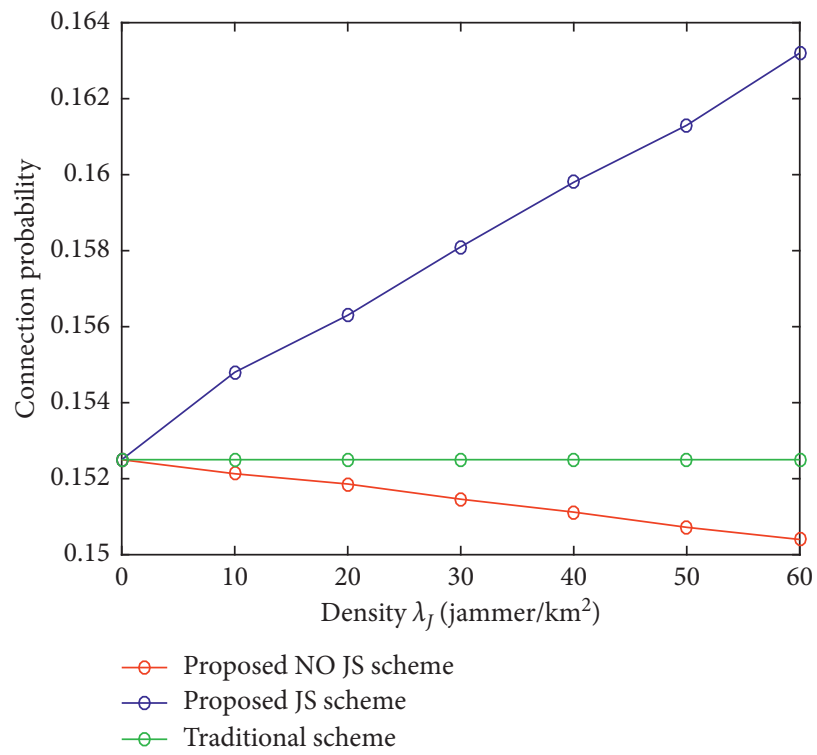

FIGURE 8: The connection probability performance of $U E_{1}$ versus jammer density $\lambda_{J}$ in three schemes.

decreases, as $\lambda_{J}$ increases. The connection probability of traditional scheme without jointing jammer remains constant. This is because the jammers whose interference on any scheduled user is stronger than a threshold are prevented from being active. When $\lambda_{J}$ increases, there are more jammers around the eavesdropper, but the interference level suffered by the legitimate user from jammers is mitigated, and the interference level remains low in the jammer selection scheme. When $\lambda_{J}=0$, the performance is the same between our proposed schemes and the traditional scheme. 


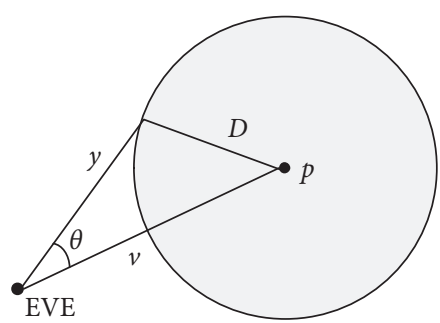

(a)

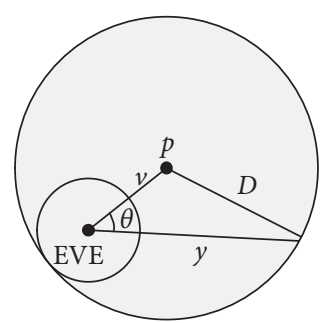

(b)

FiguRE 9: Illustration of the closest jammer hole to the eavesdropper. (a) $V \leq D$. (b) $v<D$.

\section{Conclusion}

This paper analyzes the outage and secrecy performances of NOMA network using stochastic geometry theory. The analytical expressions of outage and secrecy probabilities are derived. Compared with the "NO JS scheme" and traditional scheme (without jointing jammer), the jammer selection scheme provides a good balance between the user outage probability and secrecy probability. Simulation results show that the expressions can provide sufficient precision to evaluate the system performance. We can optimize jammer selection exclusion zone radius and NOMA power allocation to realize a secrecy transmission in future study.

\section{Appendix}

\section{A. Derivation of $L_{I_{I S \longrightarrow E}}(s)$}

To derive the $L_{I_{I S} \rightarrow E}(s)$, we use the approach in [20] (see Figure 9).

Point-p is the closest hole location to the EVE as in [20]. $V$ is the distance between the jammer and EVE. $Y$ and $v$ have a cosine-law relation: $D^{2}=y^{2}+v^{2}-2 y v \cos (\theta) . L_{I_{I S \rightarrow E}}(s)$ can be given as

$$
\begin{aligned}
L_{I_{J S \rightarrow E}}(s)= & E_{\Phi_{J S}, h}\left[-\exp \left(-s \sum_{j \in \Phi_{J S}} P_{J} h_{e, j} R_{e, j}^{-a}\right)\right] \\
= & E_{\Phi_{J}}\left(\prod_{j \in \Phi_{J} \backslash B(p, D)} L_{h_{e, j}}\left(s P_{J} R_{e, j}^{-a}\right)\right) \\
\stackrel{(c)}{=} & \exp \left(-\pi \lambda_{J} \frac{\left(s P_{J}\right)^{\delta}}{\sin c(\delta)}\right) \\
& \times E_{p}\left(\exp \left(\lambda_{J} \int_{B(p, D)}\left(1-L_{h_{e, j}}\left(s P_{J} y^{-a}\right)\right) \mathrm{d} y\right)\right) \\
= & \exp \left(-\pi \lambda_{J} \frac{\left(s P_{J}\right)^{\delta}}{\sin c(\delta)}\right) \times \int_{0}^{\infty} H(v, s) g(v) \mathrm{d} v .
\end{aligned}
$$

In step $\odot, R_{e, j}=y$ denotes the distance between selective jammers with EVE. The final step follows from the probability-generating functional of PPP $[13,19]$ and cosine-law
$D^{2}=y^{2}+v^{2}-2 y v \cos (\theta)$ and some geometry derivation [20]:

$$
H(v, s)=\left\{\begin{array}{l}
\exp \left(\int_{v-D}^{v+D} 2 y \lambda_{J e}\left(1+\frac{y^{a}}{s P_{J}}\right)^{-1} \mathrm{~d} y\right), \quad v>D, \\
\exp \left(\pi \lambda_{J}(D-v)_{2}^{2} F_{1}\left(1,1-\frac{2}{a} ; 1+\frac{2}{a} ; \frac{-(D-v)^{a}}{s P_{J}}\right)\right) \\
\times \exp \left(\int_{v-D}^{v+D} 2 y \lambda_{J e}\left(1+\frac{y^{a}}{s P_{J}}\right)^{-1} \mathrm{~d} y\right), \quad v \leq D .
\end{array}\right.
$$

where

$$
\begin{aligned}
\lambda_{\text {Je }}(y) & =\lambda_{J} \arccos \left(\frac{y^{2}+v^{2}-D^{2}}{2 y v}\right), \\
g(v) & =2 \pi \lambda_{U_{s}}\left(r_{x}, v\right) v \exp \left(-2 \pi \int_{0}^{v} \lambda_{U_{s}}\left(r_{x}, v\right) y \mathrm{~d} y\right), \\
\lambda_{U_{s}}\left(r_{x}, v\right) & =\lambda_{B}\left(1-\exp \left(-\pi\left(r_{x}+y\right)^{2} \lambda_{B}\right)\right),
\end{aligned}
$$

This completes the proof.

\section{Data Availability}

The data that support the findings of this study are available from the corresponding author upon request.

\section{Conflicts of Interest}

The authors declare that they have no conflicts of interest.

\section{Acknowledgments}

This study was supported by the Natural Science Foundation of China (Grant nos. 61801006 and 61603003), Key Laboratory of Intelligent Perception and Computing of Anhui Province, Anqing Normal University, China, Key Project on Anhui Provincial Natural Science Study by Colleges and Universities (Grant nos. KJ2017A356 and KJ2018A0361), and Natural Science Foundation of Anhui Province (Grant nos. 1608085 and 1908085MF194). 


\section{References}

[1] B. Dai, Z. Ma, Y. Luo, X. Liu, Z. Zhuang, and M. Xiao, "Enhancing physical layer security in internet of things via feedback: a general framework," IEEE Internet of Things Journal, p. 1, 2019.

[2] Z. Wang, R. F. Schaefer, M. Skoglund, M. Xiao, and H. V. Poor, "Strong secrecy for interference channels based on channel resolvability," IEEE Transactions on Information Theory, vol. 64, no. 7, pp. 5110-5130, 2018.

[3] B. Dai, Z. Ma, M. Xiao, X. Tang, and P. Fan, "Secure communication over finite state multiple-access wiretap channel with delayed feedback," IEEE Journal on Selected Areas in Communications, vol. 36, no. 4, pp. 723-736, 2018.

[4] J. Chen, Y. Liang, and M. S. Alouini, "Physical layer security for cooperative NOMA systems," IEEE Transactions on Vehicular Technology, vol. 67, no. 5, pp. 4645-4649, 2018.

[5] Z. Ding, Z. Yang, P. Fan, and H. V. Poor, "On the performance of non-orthogonal multiple access in 5G systems with randomly deployed users," IEEE Signal Processing Letters, vol. 21, no. 12, pp. 1501-1505, 2014.

[6] L. Dai, B. Wang, Y. Yuan, S. Han, C.-1. I, and Z. Wang, "Nonorthogonal multiple access for 5G: solutions, challenges, opportunities, and future research trends," IEEE Communications Magazine, vol. 53, no. 9, pp. 74-81, 2015.

[7] Y. Liu, Z. Qin, M. Elkashlan, Y. Gao, and L. Hanzo, "Enhancing the physical layer security of non-orthogonal multiple access in large-scale networks," IEEE Transactions on Wireless Communications, vol. 16, no. 3, pp. 1656-1672, 2017.

[8] G. Gomez, F. J. Martin-Vega, F. Javier Lopez-Martinez, Y. Liu, and M. Elkashlan, "Physical layer security in uplink NOMA multi-antenna systems with randomly distributed eavesdroppers," IEEE Access, vol. 7, pp. 70422-70435, 2019.

[9] Y. Tao, Y. Lei, Y. J. Guo et al., "A non-orthogonal multipleaccess scheme using reliable physical-layer network coding and cascade-computation decoding," IEEE Transactions on Wireless Communications, vol. 16, no. 3, pp. 1633-1645, 2017.

[10] C. Wang and H. M. Wang, "Opportunistic jamming for enhancing security: stochastic geometry modeling and analysis," IEEE Transactions on Vehicular Technology, vol. 65, no. 12, pp. 10213-10217, 2016.

[11] A. H. Abdel-Malek, A. M. Salhab, S. A. Zummo et al., "Power allocation and cooperative jamming for enhancing physical layer security in opportunistic relay networks in the presence of interference," Transactions on Emerging Telecommunications Technologies, vol. 28, no. 11, p. e3178, 2017.

[12] W. Tang, S. Feng, Y. Ding et al., "Physical layer security in heterogeneous networks with jammer selection and full-duplex users," IEEE Transactions on Wireless Communications, vol. 16, no. 12, pp. 7982-7995, 2017.

[13] H. S. Jo, Y. J. Sang, P. Xia et al., "Heterogeneous cellular networks with flexible cell association: a comprehensive downlink SINR analysis," IEEE Transactions on Wireless Communications, vol. 11, no. 10, pp. 3484-3495, 2011.

[14] A. He, L. Wang, M. Elkashlan et al., "Spectrum and energy efficiency in massive MIMO enabled HetNets: a stochastic geometry approach," IEEE Communications Letters, vol. 19, no. 12, pp. 2294-2297, 2015.

[15] H. Elsawy, E. Hossain, and M. Haenggi, "Stochastic geometry for modeling, analysis, and design of multi-tier and cognitive cellular wireless networks: a survey," IEEE Communications Surveys Tutorials, vol. 15, no. 3, pp. 996-1019, 2013.

[16] M. D. Renzo, "Stochastic geometry modeling and analysis of multi-tier millimeter wave cellular networks," IEEE
Transactions on Wireless Communications, vol. 14, no. 9, pp. 5038-5057, 2015.

[17] Y. Sun, Z. Ding, X. Dai et al., "On the performance of network NOMA in uplink CoMP systems: a stochastic geometry approach," 2018, http://arxiv.org/abs/1803.00168.

[18] Z. Zhang, H. Sun, and R. Q. Hu, "Downlink and uplink nonorthogonal multiple access in a dense wireless network," IEEE Journal on Selected Areas in Communications, vol. 35, no. 12, pp. 2771-2784, 2017.

[19] M. Haenggi, Wireless Security and Cryptography: Specifications and Implementations, Cambridge University Press, Cambridge, UK, 2012.

[20] Z. Yazdanshenasan, H. S. Dhillon, M. Afshang, and P. H. J. Chong, "Poisson hole process: theory and applications to wireless networks," IEEE Transactions on Wireless Communications, vol. 15, no. 11, pp. 7531-7546, 2016. 


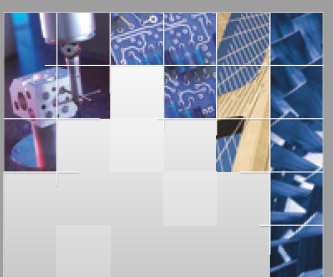

\section{Enfincering}
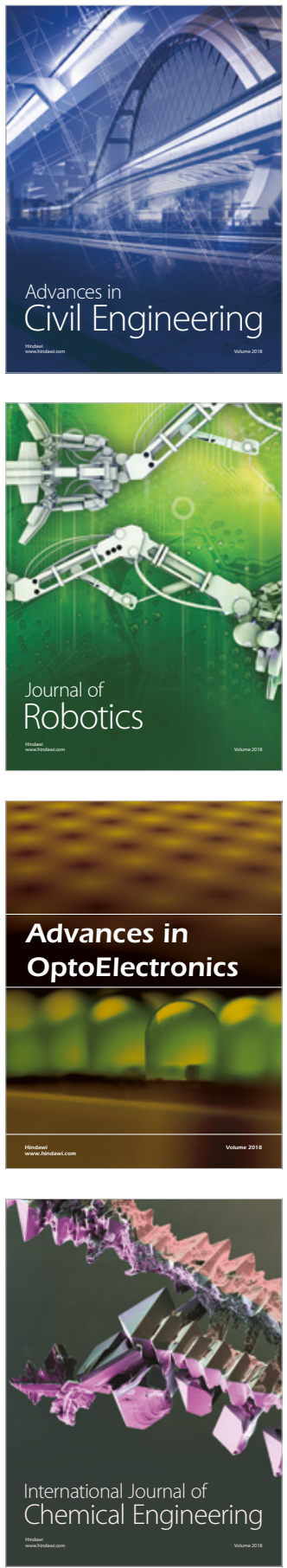

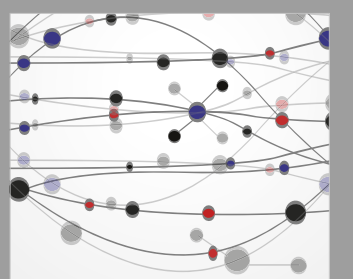

\section{Rotating \\ Machinery}

The Scientific World Journal

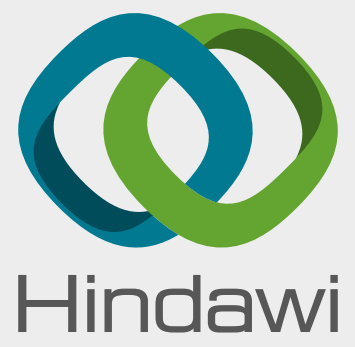

Submit your manuscripts at

www.hindawi.com
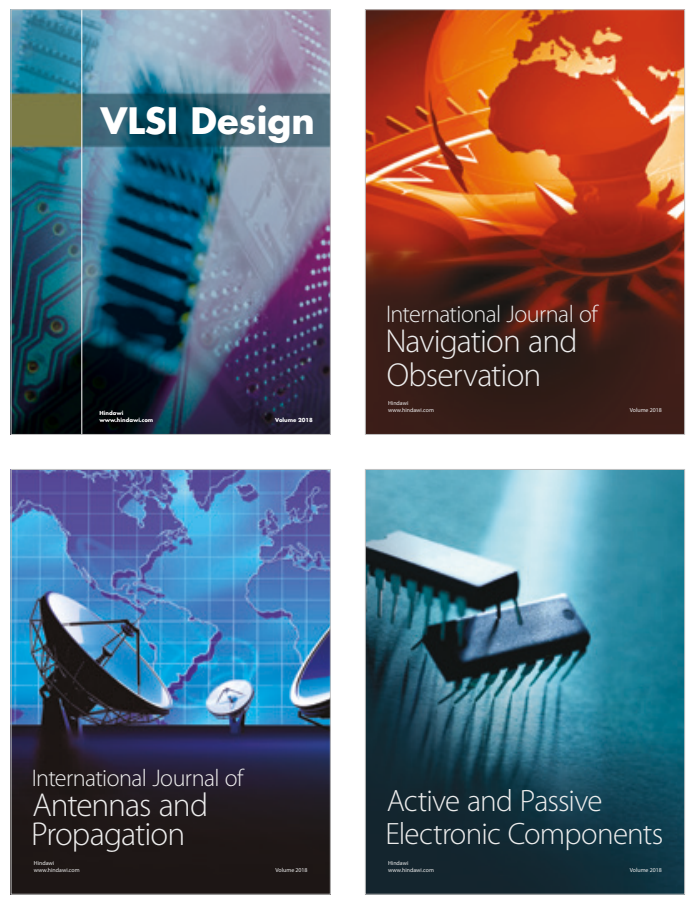
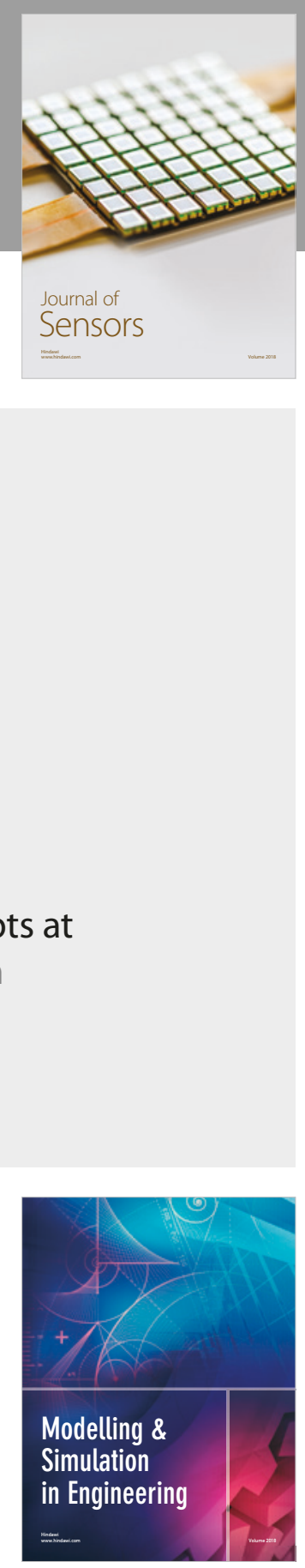

\section{Advances \\ Multimedia}
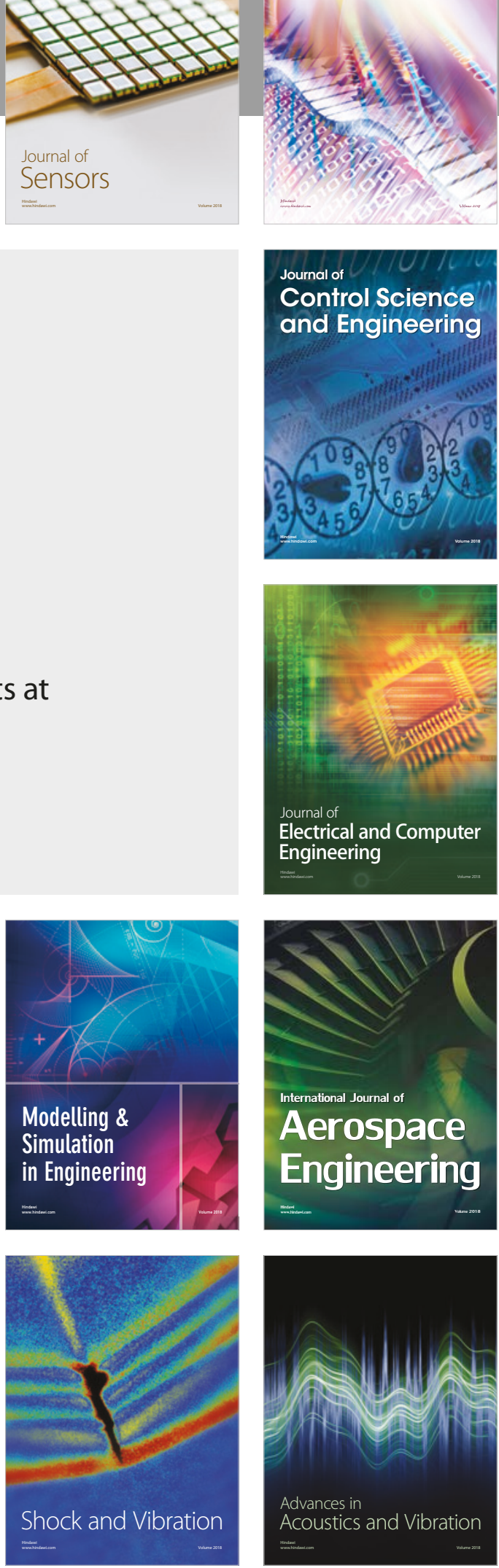